\title{
Effect of Concentration Level on Mass Transfer Rates
}

\section{E. WESTKAEMPER and ROBERT R. WHITE}

University of Michigan, Ann Arbor, Michigan

Carbon tetrachloride was evaporated into a stream of air over ranges of gas concentration from 0 to 0.70 mole fraction carbon tetrachloride, Reynolds number from 600 to 15,000, and Schmidt number from 0.23 to 1.17 . The data were correlated by an equation and also by a computer solution to the differential equation describing mass transfer from values of eddy viscosity and eddy diffusivity obtained from the literature.

Investigations of mass transfer have resulted in the development of methods for correlation and prediction of mass transfer rates in a variety of situations. The effect of Reynolds number, Schmidt number, and similar variables has been analyzed for many systems; however, the effect of concentration level has received little attention.

Various terms in mass transfer calculations contain concentration level as a variable. For example, the $j$ factor for mass transfer has been defined by Colburn (2) as

$$
j_{D}=\frac{k_{g} p_{B m} S c^{2 / 3}}{G_{m}}
$$

where $p_{B m}$ is a log-mean concentration term.

Experimental studies on the effect of concentration level on mass transfer rates have been meager. Cairns and Roper (1) studied the concentration-level variable in the air-water system and correlated their data by the following equation:

$$
\begin{aligned}
\frac{k_{g} R T d}{D_{v}}\left(\frac{p_{B_{m}}}{P}\right)^{0.83}(S c)^{-0.44} & =0.021 R e^{0.83}
\end{aligned}
$$

the ratio $\left(p_{B m} / P\right)$ was varied from 0.15 to 0.99 by Cairns and Roper, and concentration level enters their correlation as an important variable.

The objectives of the present study were to collect mass transfer rate data in the air-carbon tetrachloride system over a wide range of concentration level and to study various methods of correlating the data.

\section{EXPERIMENTAL WORK}

Liquid carbon tetrachloride was evaporated from a plane surface in the bottom of a rectangular duct into a turbulent air stream passing through the duct.

Gas concentrations were varied from 0.00 to 0.70 mole fraction carbon tetrachloride. Reynolds number varied from 600 to 14,700 and the Schmidt number from 0.23 to 1.17 . Gas temperatures varied from 68 to $91^{\circ} \mathrm{C}$., and all experiments were conducted at atmospheric pressure. The ratio $\left(p_{B m} / P\right)$ varied from 0.29 to 0.81 .

The test section is shown in Figure 1 .

L. E. Westkaemper is at present with Rohm and Haas Company, Pasadena, Texas.
Liquid enters the section through a 1-in. pipe at $A$ and is distributed over the test area by the perforated plate $P P$. The plate is perforated by $1 / 16-\mathrm{in}$. holes spaced on
$1 / 2$-in. equilateral triangle centers. Liquid leaves the test section at overflow weirs $B$ and $C$ and flows into a holding tank. Testsection details are given in Figure 2. The

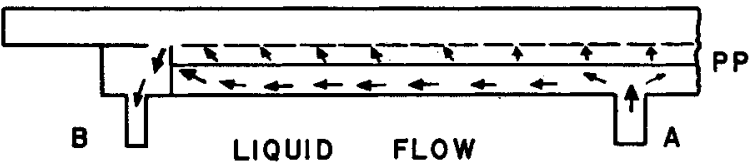

Fig. 1. Test section.

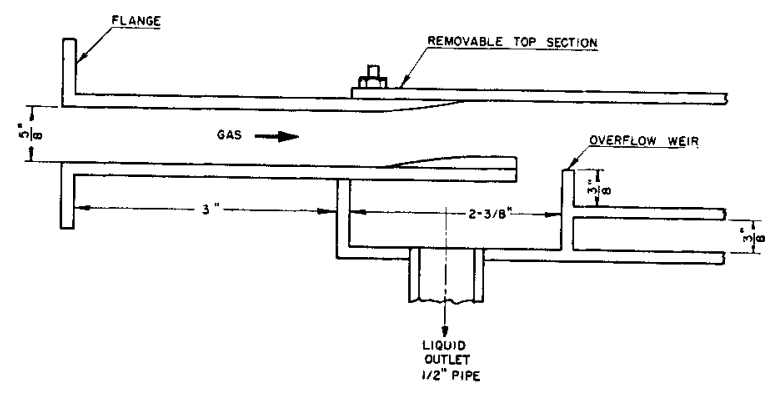

Fig. 2. Test section details; $1 / 8$ in. sheet steel throughout inlet cross section and cross section for gas flow over liquid: $5 / 8$ - by $41 / 16$-in. length, 48 in. between weirs.

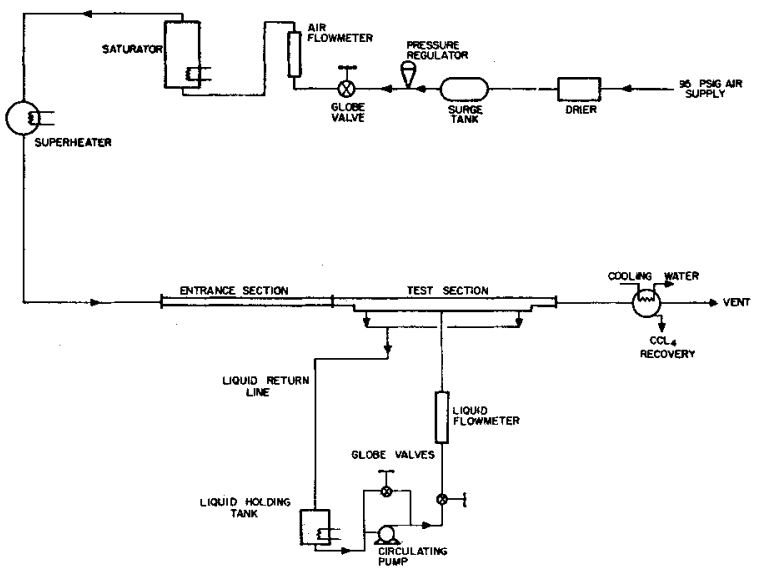

Fig. 3. Schematic flow sheet. 
wetted area of the test section is $1.35 \mathrm{sq} . \mathrm{ft}$.

The remaining experimental apparatus is identified in the flow sheet shown in Figure 3 . The entrance section preceding the test section is a 36-in. length of rectangular duct of cross section $41 / 16$ by $5 / 8$ in., which is identical to the cross section for gas flow above the liquid in the test section.

The saturator shown in Figure 3 is a 36-in. length of 12-in. steel pipe provided with two 1,500-watt immersion heaters and a thermoregulator. A perforated plate in the base distributes incoming air through the liquid carbon tetrachloride in the saturator, and the air leaves saturated with carbon tetrachloride vapors.

The bottom of the liquid holding tank is made from 12-in. steel pipe. A sight glass was placed on a length of 2-in. pipe forming the top of the tank to increase the sensitivity of volume readings.

A steel top was used on the test section during the experimental runs. However, a glass top was used in preliminary runs to observe the maximum gas velocities with stable gas-liquid interfaces. It was observed that ripples appeared on the liquid surface when gas velocities exceeded 10 to $12 \mathrm{ft}$. $/ \mathrm{sec}$. A small increase in gas velocity at this level caused the ripples to grow and become unstable; i.e., the ripples grew until entrainment of liquid in the gas resulted. When the surface was unstable, the entrained liquid was carried out into the exit lines, and thus the pressure drop through the vent lines was more than doubled.

In the evaporation experiments the pressure drop through the vent lines was carefully observed during the start-up

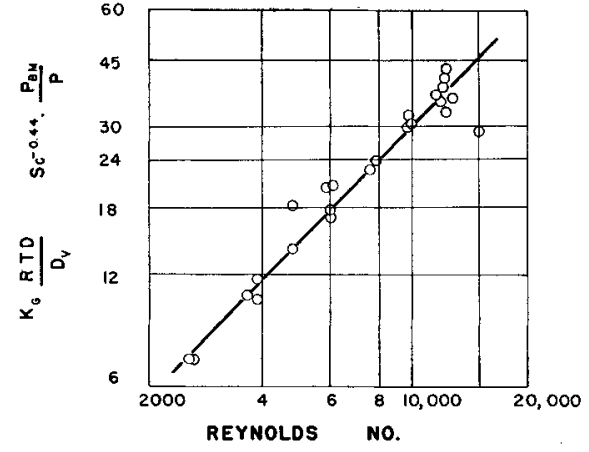

Fig. 4. Correlation of the data with a concentration term.

period, and if it was steady as the gas velocity was increased to operating conditions, the run was continued.

The compositions reported as experimental data were determined as follow. The inlet composition was calculated from the temperature and pressure of the gas by assuming that it was at its dew point when it left the saturator. The validity of this assumption was verified in preliminary runs by an analysis of the gas made by quantitative charcoal absorption of the carbon tetrachloride in a measured gas sample.

The change in the composition of the gas passing through the test section was relatively small; therefore error in gas analysis is magnifed when the change in composition is taken. For this reason, it was felt that the exit-gas composition would best be determined by calculation from the measured evaporation rate, the inlet-gas rate, and the inlet-gas composition.

Check runs showed that evaporation rates could be reproduced within $10 \%$, as may be seen by comparing runs 12 and 14 , 2 and 4 , and 18 and $18 a$ in Table 1 .

Additional experimental details and data are available elsewhere $(6)$.

\section{CORRELATION OF THE DATA}

\section{Dimensionless Groups}

Various methods of correlating the data have been used by previous investigators. For convenience in comparing the results with those from other sources, the data were correlated by plotting $\left[\left(k_{q} R T d\right) / D_{v}\right] \cdot p_{B m} / P \cdot S c^{n-1}$ as a function of Reynolds number. This term is equal to $(j)$. (Re) where $j=\left(k_{g} p_{B m} S c^{n}\right) / G_{m}$. Figure 4 shows a correlation of the data for this investigation. The equation of the line shown is

$$
\frac{k_{g} R T d}{D_{v}} \cdot \frac{p_{B m}}{P} \cdot S c^{-0.44}=0.0014 R e^{1.08}
$$

The standard deviation of the error is $16 \%$.

Figure 5 shows the data plotted in a manner similar to that of Figure 4, except that the term $\left(p_{B m} / P\right)$ has been omitted from the ordinate. The equation of the line shown is

$$
\frac{k_{g} R T d}{D_{v}} \cdot S c^{-0.44}=0.00012 R e^{1.42}
$$

The standard deviation of the error is $15 \%$. The ordinate of Figure 5 is equal to $(j)(R e)$ when $j=\left(k_{g} P S c^{0.56}\right) / G_{m}$.

Data taken by Cairns and Roper for the evaporation of water into air in a wetted-wall column may be plotted in a manner similar to that shown in Figure 4. A plot of Cairns and Roper's data is shown in Figure 6. The equation of the line shown is

$$
\frac{k_{g} R T d}{D_{v}} \cdot \frac{p_{B m}}{P} \cdot S c^{-0.44}=0.061 R e^{0.67}
$$

The standard deviation of the error is $21 \%$. Cairns and Roper improved the correlation of their data as shown in Figure 7 and used the following equation:

$$
\begin{aligned}
\frac{l_{g} R T d}{D_{v}}\left(\frac{p_{B m}}{P}\right)^{0.83} S c^{-0.44} & \\
& =0.021 R e^{0.83}
\end{aligned}
$$

Here the standard deviation of the error is reduced to $10 \%$. If the form shown by Equation (3) is used to correlate Cairns and Roper's data, the correlation is quite poor.

Equations (1) to (4) were determined from the data by the method of least squares.

Equations (2) and (3) show that the definition of the $j$ factor influences the exponent of the Reynolds number in the 
correlation. An exponent of 1.08 appears on the Reynolds number in Equation (2), where $j=\left(k_{v} p_{B m} S c^{0.56}\right) / G_{m}$.

An exponent of 1.42 appears on the Reynolds number in Equation (3), where $j=\left(k_{g} P S c^{0.56}\right) / G_{m}$.

Cairns and Roper were able to focus attention on the $\left(p_{B m} / P\right)$ term, since the Schmidt number was held constant in their research. The $\left(p_{B m} / P\right)$ must be included in the correlation of their data. The air-carbon tetrachloride data of this paper may also be correlated by use of the $\left(p_{B m} / P\right)$ term, as is shown by Equation (2). Hence the form shown by Equations (2) and (4) seems to be the more general, as the form given by Equation (3) will not correlate the data of Cairns and Roper. Since Equations (2) and (4) correlate the data of this investigation with deviations of 16 and $15 \%$ respectively, Equation (1) used by Cairns and Roper also will correlate the data as well.

\section{Differential Equation}

In the case of molecular transport processes, the transfer rates for momentum, heat, and mass are given by

$$
\begin{gathered}
\tau=\mu \frac{d u}{d y} \\
q=-k \frac{d T}{d y} \\
N_{A}=-D_{v} \frac{d c}{d y}
\end{gathered}
$$

Here the conductance terms are viscosity, thermal conductivity, and molecular diffusivity. The potential terms are the gradients of velocity, temperature, and concentration. Each potential term is in differential form.

Similar treatment is made in the case of turbulent transfer processes. Expanding the molecular transfer equations

$$
\begin{aligned}
\tau & =\mu \frac{d u}{d y}+\rho \epsilon_{m} \frac{d u}{d y} \\
q & =-k \frac{d T}{d y}-\rho c_{p} \epsilon_{c} \frac{d T}{d y} \\
N_{A} & =-D_{\vartheta} \frac{d c}{d y}-E \frac{d c}{d y}
\end{aligned}
$$

The foregoing equations serve to define $\epsilon_{m}$, eddy viscosity; $\epsilon_{c}$, eddy conductivity; and $E$, eddy diffusivity. In each case the units are (distance) ${ }^{2}$ per unit time. The definitions take this form in the hope that the similarity of the definitions will result in simple relationships between eddy properties; that is, it is hoped that the eddy properties will be equal or proportional to one another.

In this work the primary interest is in the mass transfer rate equation (10). From this equation and a material balance, the equation for turbulent mass transfer is derived:

$$
\begin{aligned}
\frac{d c}{d t}= & \frac{\partial}{\partial x}\left(D_{v}+E\right) \frac{\partial c}{\partial x}-\frac{\partial(u c)}{\partial x} \\
& +\frac{\partial}{\partial y}\left(D_{v}+E\right) \frac{\partial c}{\partial y}-\frac{\partial(v c)}{\partial y} \\
& +\frac{\partial}{\partial z}\left(D_{v}+E\right) \frac{\partial c}{\partial z}-\frac{\partial(w c)}{\partial z}
\end{aligned}
$$

Equation (11) relates gas concentration to time, gas velocity, eddy diffusivity, molecular diffusivity, and position in the system. Under steady state conditions for turbulent gas flow between parallel plates in the $x$ direction and mass transfer in the $y$ direction normal to the plates, $d c / d t=0$ and $w=0$. For the experiments of this investigation $\partial u / \partial x, \partial / \partial x$ $\left[\left(D_{v}+E\right)(\partial c / \partial x)\right]$, and $v$ are negligible, and so Equation (11) simplifies to

$$
u \frac{\partial c}{\partial x}=\frac{\partial}{\partial y}\left[\left(D_{v}+E\right) \frac{\partial c}{\partial y}\right]
$$

In the experimental work only small changes in gas composition occurred in the 4-ft. length of the test section. Temperature changes in the gas were small, as were changes in the gas volume. Hence the assumptions that $\partial u / \partial x=0$ and $(\partial / \partial x)\left[\left(D_{v}+E\right)(\partial c / \partial x)\right]=0$ are justifiable.

The assumption that $v=0$, however, is valid only over certain ranges of operation. This assumption was necessary in separating the variables in Equation (11). The comparison of the evaporation rates calculated by use of Equation (12) with the experimental rates is shown in Table 2. Mathematical details are available elsewhere (6). The calculated values are seen to be in error at low Reynolds numbers. Previous workers have shown that the presence of a velocity normal to the wall is important in momentum transfer studies.

Yuan and Finkelstein (9) have shown that the presence of a velocity normal to the surface $1 / 100$ as great as the mainstream velocity increases the friction by $85 \%$. The actual value of $v$ in this work is given by $N_{A} / c_{t}$, the moles transferred per unit time per unit area divided by the total gas concentration in moles per unit volume. When main-stream velocity is lowered, this term becomes increasingly important for a given evaporation rate. Consequently, it is felt that the poor correlation of the data at low Reynolds numbers is caused by neglecting $v$.

The solution of Equation (12) requires that velocity $u$ and eddy diffusivity $E$ be known as a function of position. The variation of velocity with distance from the wall has been measured by Corcoran et al. (3) for air passing between parallel plates. The velocity distribution was measured in an isothermal as well as in a nonisothermal system. Temperature differences between the plates of $20^{\circ} \mathrm{F}$. did not appreciably alter the velocity distribution observed. Dimensionless ve-

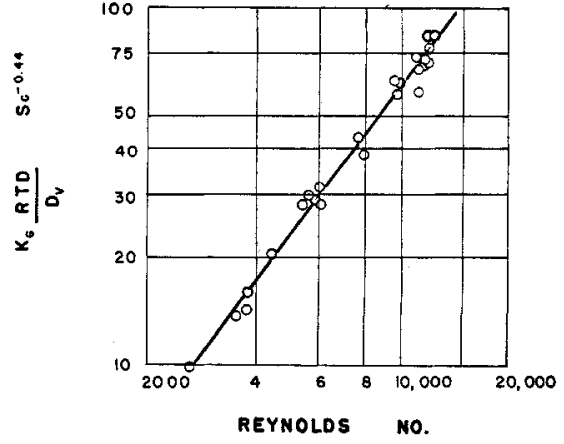

Fig. 5. Correlation of the data without a concentration term.

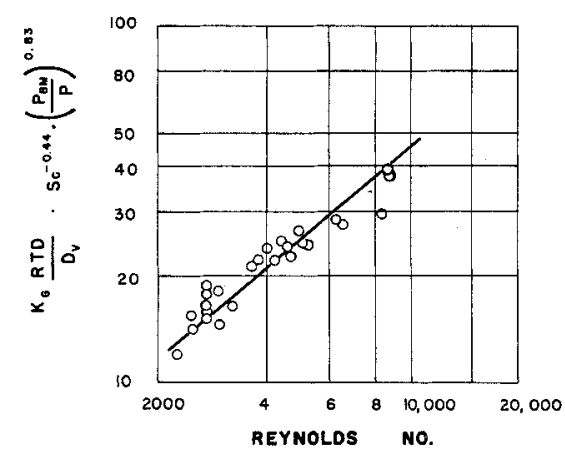

Fig. 6. Correlation of Cairns and Roper.

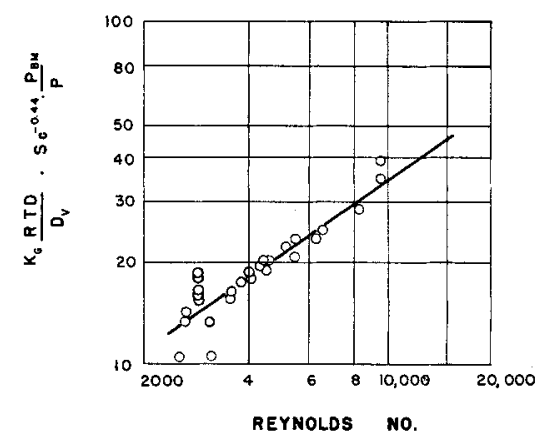

Fig. 7. Data of Cairns and Roper.

locity based on these data is shown in Figure 8.

Since the variation of eddy diffusivity with distance from the wall has not been reported in the literature, this information must be obtained indirectly.

It was noted that eddy viscosity, eddy conductivity, and eddy diffusivity were defined by Equations (8) to (10) in the hope that these quantities would be equal or proportional. Various researchers have conducted experiments to determine the relationship between the eddy properties.

Woertz (8) measured concentration distribution and mass transfer rates of water through air between parallel plates. Eddy-diffusivity values for the center region between the plates were obtained by differentiation of the concentration data and use of Equation (10).

Woertz also measured pressure drop and velocity distribution while the mass transfer process took place. Eddy- 


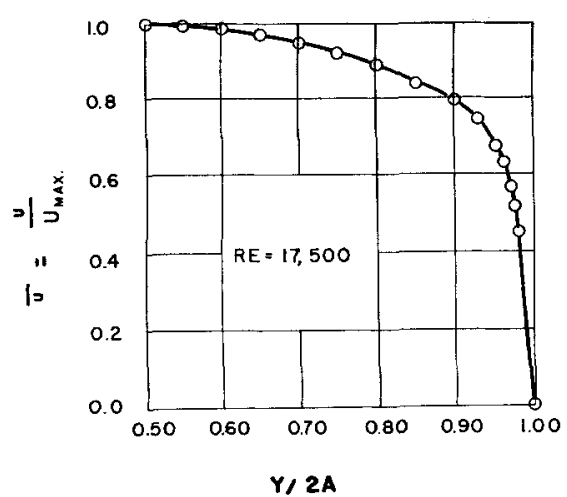

Fig. 8. Velocity distribution between parallel plates, (3).

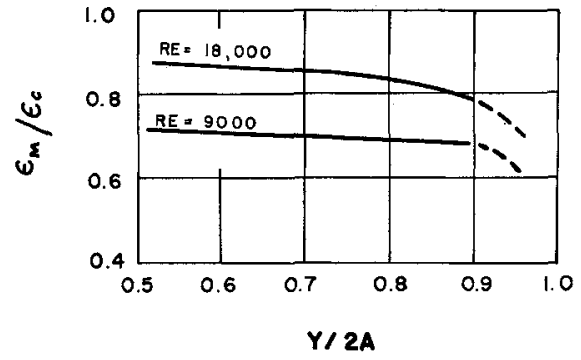

Fig. 9. Ratio of eddy viscosity to eddy conductivity, ( 3 ).

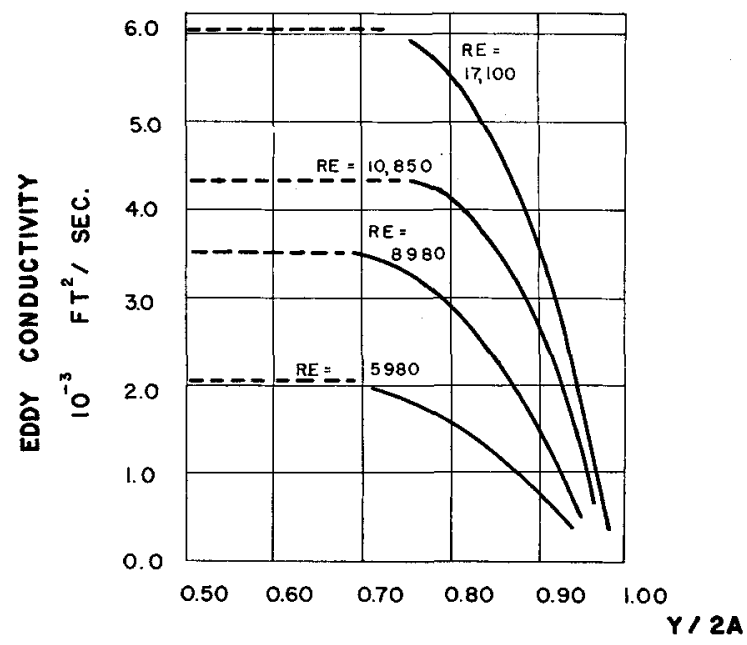

Fig. 10. Eddy conductivity values (3).

Reynolds numbers. This comparison is shown in Figure 9, where the ratio of eddy viscosity to eddy conductivity is plotted vs. distance from the wall with parameters of Reynolds number.

There has been no experimental study relating eddy conductivity to eddy diffusivity; however, a relationship may be inferred from the studies of Sage and Woertz. Figure 9 shows that the ratio of eddy viscosity to eddy conductivity is approximately 0.7 for the center of the duct at a Reynolds number of 10,000. Hence,

$$
\epsilon_{m} / \epsilon_{c}=0.7
$$

The work of Woertz showed that $\epsilon_{m} / E=$ 0.62 and hence

$$
\epsilon_{c} / E=1.1 \approx 1
$$

Equation (15) suggests that eddy conductivities may be taken as equal to eddy diffusivities when these quantities are defined by Equations (9) and (10). The units in each case are (distance) ${ }^{2}$ per unit time. Figure 10 shows the eddy conductivity values calculated by Sage for air. The characteristic diameter in the Reynolds number used by Sage was twice the hydraulic radius, and this basis is used in Figure 10. Equation (15) and Figure 8 were used to calculate eddy diffusivity values for air from eddy conductivity values for air.

In this research, however, the gas was not air, but a mixture of air and carbon tetrachloride. Consequently, a method of predicting the effect of composition on eddy diffusivity must be used. As suggested by Deissler (4), this was done with the following expression:

$$
\frac{\text { eddy diffusivity }}{\text { kinematic viscosity }}=\text { constant }
$$

viscosity values for the center region between the plates were obtained by calculating the shear stress from the pressure drop, differentiating the velocity data, and using Equation (8).

Woertz found that the ratio of the eddy viscosity to eddy diffusivity in the center of the duct for Reynolds numbers up to 60,000 was 0.62 ; i.e.,

$$
\epsilon_{m} / E=0.62
$$

Further study of eddy properties was made by Sage and associates (3), who considered the relation between eddy viscosity and eddy conductivity.

Sage determined eddy viscosity for air passing between parallel plates by use of Equation (8), as shown above. Sage also measured heat flux and temperature distribution. Eddy conductivity values were obtained by differentiating the temperature data and using Equation (9).

Sage obtained more detailed distributions of velocity and temperature than

\begin{tabular}{|c|c|c|c|c|c|c|}
\hline Run & $(R e)$ & $\begin{array}{c}\bar{c} \\
\text { calc. }\end{array}$ & $\begin{array}{c}\bar{c} \\
\text { exp. }\end{array}$ & $\begin{array}{c}N \\
\text { calc. }\end{array}$ & $\begin{array}{c}N \\
\text { exp. }\end{array}$ & $\%$ Dev. \\
\hline 2 & 11,900 & 0.614 & 0.600 & 0.0110 & 0.0123 & -10.6 \\
\hline 4 & 12,200 & 0.608 & 0.595 & 0.0113 & 0.0115 & -1.7 \\
\hline 6 & 9,800 & 0.598 & 0.627 & 0.0107 & 0.0099 & 8.1 \\
\hline 7 & 9,500 & 0.561 & 0.615 & 0.0101 & 0.0086 & 17.4 \\
\hline 8 & 11,800 & 0.623 & 0.687 & 0.0093 & 0.0075 & 24.0 \\
\hline 9 & 12,200 & 0.631 & 0.626 & 0.0077 & 0.0077 & 0.0 \\
\hline 11 & 11,600 & 0.629 & 0.635 & 0.0083 & 0.0081 & 2.5 \\
\hline 14 & 5,790 & 0.190 & 0.690 & 0.0155 & 0.0050 & 210 \\
\hline 16 & 3,750 & 0.507 & 0.757 & 0.0052 & 0.0025 & 108 \\
\hline 17 & 5,570 & 0.221 & 0.682 & 0.0139 & 0.0051 & 172 \\
\hline 18 & 2,690 & 0.227 & 0.806 & 0.0080 & 0.0022 & 264 \\
\hline 21 & 4,470 & 0.497 & 0.707 & 0.0085 & 0.0047 & 81 \\
\hline 28 & 13,100 & 0.675 & 0.633 & 0.0039 & 0.0044 & -11.4 \\
\hline 29 & 14,700 & 0.670 & 0.684 & 0.0080 & 0.0079 & 1.9 \\
\hline 30 & 4,690 & 0.865 & 0.530 & 0.0005 & 0.0018 & -73 \\
\hline 31 & 7,490 & 0.576 & 0.612 & 0.0036 & 0.0031 & 17.4 \\
\hline
\end{tabular}
did Woertz. Hence Sage compared eddy viscosity to eddy conductivity for various
Table 2. Comparison of Computed and Experimental Results.

$N$, evaporation rate, std. cu. ft./sec.

$\bar{c}$, dimensionless outlet concentration, $\left(c-c_{w}\right) /\left(c_{0}-c_{w}\right)$

$\%$ Dev, $(\exp . N$ - calc. $N) /(\exp . N)(100)$

(Re), Reynolds number, characteristic radius equal to the clearance between plates 
A similar relation was used by Woertz, who found that the product $(E \rho)$ was the same for air, carbon dioxide, and helium at the same Reynolds number. Since the viscosities of air, carbon dioxide, and helium do not vary greatly, Woertz could have used the product $(E \rho / \mu)=$ $(E / \nu)$ as Deissler suggested.

Equation (16) was used to calculate eddy diffusivity in air-carbon tetrachloride as a function of position from the eddy diffusivities in pure air, except for the value of eddy diffusivity at the liquid surface. The viscosity of the aircarbon tetrachloride mixtures was calculated by use of the correlation and data of Wilke and Bromley (7).

A final calculation was necessary to obtain the eddy diffusivity at the liquid surface. Since one of the "parallel plates" of this research was actually a liquid surface, it could not be considered as a fixed surface. The drag of the gas stream on the liquid surface causes a surface velocity in the liquid, as shown by Tek (5); hence the gas velocity does not drop to zero at the liquid surface but instead drops to a value equal to the surface velocity of the liquid.

Tek has developed equations which may be used to calculate the liquid surface velocity and the surface shear from the main-stream gas velocity and the properties of the liquid. This calculation involves the use of Equations (17) and (18), which were developed by Tek (5).

$$
u_{s}=\frac{(d h / d x) a_{l}{ }^{2} g_{c}}{6 \nu_{1}}
$$

where $(d h / d x)=k_{T} U_{a v g}{ }^{2}=$ surface inclination due to gas velocity,

and

$$
g_{c} \tau_{s}=\frac{4 \mu_{l} u_{s}}{a_{l}}
$$

Eliminating $\left(u_{s}\right)$ from Equations and (18) gives

$$
\tau_{s}=\frac{4}{6} a_{l} U_{a v g}^{2} k_{T} \frac{\mu_{l}}{\nu_{l}}
$$

Equation (19) may be used to calculate the shear stress at the liquid surface, which is also given by Equation (8). For the case of the liquid surface, in units consistent. with Equation (19), Equation (8) becomes

$$
g_{c} \tau_{s}=\left(\mu+\epsilon_{m} \rho\right)_{*}\left(\frac{d u}{d y}\right)_{s}
$$

Combining (19) and (20) and solving for $\left(\mu+\epsilon_{m} \rho\right)_{s}$ gives

$$
\begin{aligned}
\left(\mu=\boldsymbol{\epsilon}_{m} \rho\right)_{s} & =\frac{g_{c} \frac{4}{6} a_{l} U_{a v g}{ }^{2} k_{T}\left(\mu_{l} / \nu_{l}\right)}{d u / d y} \\
& =\frac{2 a_{1} g_{c} U_{a v g}{ }^{2} k_{T} \rho_{l}}{3(d u / d y)_{s}}
\end{aligned}
$$

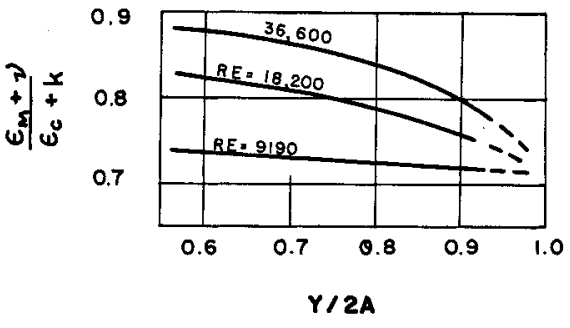

Fig. 11. Ratio of total viscosity to total conductivity $(3)$.

and

$$
\left(\nu+\epsilon_{m}\right)_{s}=\frac{2 a_{l} g_{c} U_{a v g}{ }^{2} k_{T} \rho_{l}}{3 \rho(d u / d y)_{s}}
$$

Equation (22) gives the "total" viscosity $\left(\nu+\epsilon_{m}\right)$ at the surface as a function of the average velocity $U_{a v g}$ and the velocity gradient at the surface $(d u / d y)_{s}$.

It was assumed that the velocity gradient at the liquid surface was equal to the velocity gradient at the wall in Sage's experiments. Accordingly, the values of $(d u / d y)$, were calculated by differentiating the velocity data shown in Figure 8 and the total viscosities calculated from Equation (22).

Actually the velocity gradient at the liquid surface will be somewhat less than the velocity gradient at a fixed boundary, and therefore the values of total viscosity obtained by the foregoing procedure are low. As a result, the values of eddy conductivity or eddy diffusivity derived from the eddy viscosity will also be low and will tend to decrease the rate calculated from Equation (12)

Sage reports values of the ratio of total viscosity $\left(\nu+\epsilon_{m}\right)$ to total conductivity $\left[k /\left(\rho c_{p}\right)+\epsilon_{c}\right]$, and these are shown as a function of Reynolds number and position in Figure 11. Thus Figure 11 can be used to evaluate the total conductivity at the surface from the total viscosity calculated from Equation (22). The total diffusivity at the surface was taken as equal to the total conductivity at the surface.

This step is somewhat inconsistent with the use of the data of Woertz and Sage to show that $\epsilon_{c} / E=1$. Consistency would require that $\left(k / \rho c_{p}\right)$ be subtracted from total conductivity values to give eddy conductivity and eddy diffusivity. Total diffusivity would be obtained for use in Equation (12) by adding $D_{v}$ to the eddy diffusivity.

However the equation $\epsilon_{c} / E=1$ is verified only for the center region of gas flow, and the approximation of taking total conductivity equal to total diffusivity is probably as accurate as assuming that $\epsilon_{c} / E=1$ throughout the flow domain. Once a total diffusivity at the liquid surface is known, it may be used as a limiting value for the total diffusivities (6) in the main gas stream, as shown in Figure 12.

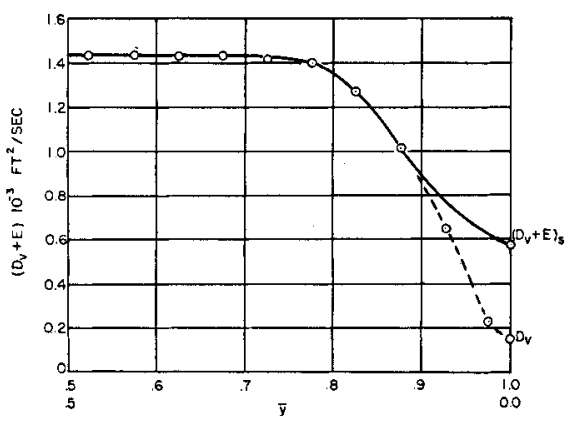

Fig. 12. Total diffusity values for run 6 .

The surface velocity of the gas was not high enough $(\bar{u} \approx 0.1)$ to alter greatly the velocity profile shown in Figure 8. As this velocity distribution was approximated in a computer solution by a step function, accuracy was not improved by adding the correction at the wall.

An electronic differential analyzer was used to solve the ordinary differential equations (6) resulting from the separation of variables in Equation (12).

A summary of the outlet concentrations calculated by computer solution of Equation (12) is shown in Table 2, which also shows the experimental outlet concentration, which was calculated from the inlet-gas concentration, the inlet-gas rate, and the evaporation rate.

Table 2 shows good agreement between the calculated and experimental evaporation rates for Reynolds numbers over 7,000 . The average deviation is $10.5 \%$ for these runs. Even though numerous approximations must be made in using Equation (11) to correlate mass transfer data, the outlet concentrations predicted by this equation agree closely with the experimental concentrations for Reynolds numbers over 7,000 , as seen in Table 2 . No arbitrary constants are used in the equation at any point. This suggests that Equation (11) may prove as useful in mass transfer as the Fourier-Poisson equation has in heat transfer.

Certain predictions may be made concerning the effect of concentration level on mass transfer by examination of Equation (12). In addition to the terms containing concentration gradient, this equation contains the term $\left(D_{v}+E\right)$, which is a function of concentration level. This term depends on concentration level because of the variation of $E$ with concentration, as has been discussed.

Hence Equation (12) suggests that for a system in which there is no change in eddy diffusivity with concentration, there would be no effect of concentration level. Also if (eddy diffusivity)/(kinematic viscosity) $=$ constant, a mixture with a kinematic viscosity independent of concentration should also have an eddy diffusivity independent of concentration.

The air-water system is one which has a gas kinematic viscosity largely inde- 
Table 3. Correlation of Data of Cairns and Roper with Equation (12)

$\begin{array}{rcc}\text { Run } & \text { Reynolds No. } & \begin{array}{c}\text { Gas } \Delta T, \\ { }^{\circ} \mathbf{F} .\end{array} \\ 4 & & \mathbf{2 5 1} \\ 9 & 6520 & \mathbf{2 5 4} \\ 11 & 9095 & 342 \\ 18 & 3273 & 315\end{array}$

Outlet concentration, $\bar{c}$ Equation (12) Cairns and Roper

$\begin{array}{ll}0.29 & 0.43 \\ 0.26 & 0.38 \\ 0.15 & 0.39 \\ 0.21 & 0.31\end{array}$

pendent of concentration. At $212^{\circ} \mathrm{F}$. the kinematic viscosities of air and steam differ by only $7 \%$. This system would be expected to show little effect of concentration level on mass transfer rates.

Extensive data in the air-water system have been taken by Cairns and Roper. While these data clearly require a concentration variable $\left(p_{B m}\right)$ in their correlation, this apparent contradiction of Equation (12) may be explained. Cairns and Roper operated a wetted-wall column in their investigation, and the temperature of the gas passing through the column changed by as much as $340^{\circ} \mathrm{F}$. Furthermore, the inlet-gas temperatures varied from run to run. Hence there was a kinematic viscosity change, caused by temperature change. The variation in the kinematic viscosity of the inlet gas for various runs was over $30 \%$ and of the gas passing through the test section was as high as $80 \%$. Most of the data taken by Cairns and Roper were for Reynolds numbers less than 7,000, where Equation (12) is not satisfactory. Nevertheless, four runs of Cairns and Roper were correlated by Equation (12) with the differential analyzer. Each calculated dimensionless outlet concentration for these runs was lower than that reported by Cairns and Roper (Table 3). Since the dimensionless concentration $\vec{c}=(c-$ $\left.c_{w}\right) /\left(c_{0}-c_{w}\right)$, the mass transfer rate predicted by concentrations from Equation (12) would be high in each case. In the derivation of Equation (12) it was assumed that the eddy diffusivity was independent of $x$. The large change in kinematic viscosity and eddy diffusivity in the direction of flow in the work of Cairns and Roper makes it unlikely that Equation (12) is adequate to correlate their data.

If the air-water system is to be used to study the effect of concentration level, the temperature drop in the gas must be held at a minimum. This suggests that an apparatus similar to that used in this study might prove more successful than a wetted-wall column, where control of gas temperature is difficult.

\section{CONCLUSION}

In summary, the facts that data of this investigation may be correlated equally well by Equations (1), (2), and (3) and that the data of Cairns and Roper may be correlated best by Equation (1) and less satisfactorily by Equation (4), which is similar in form but not in coefficients to Equation (2), lead one to suspect that functions of this type between the dimensionless groups may be too simple to represent adequately mass transfer data over a wide range in the level of concentration of the transferring component.

In a sense this is corroborated by the relatively successful result of applying the mass equation in differential form to the data correlation even though a considerable number of assumptions and the use of data on eddy properties from many sources were involved. Of particular interest is the fact that the correlation by the differential equation begins to fail exactly where it might be expected to fail at low flow rates, or Reynolds numbers less than 7,000 , where the bulk motion normal to the axis of flow and in the direction is no longer negligible.

This suggests that, as further research makes available velocity distributions in turbulent-flow regimes of various geometries, the use of computing machines will make feasible the correlation and application of mass and heat transfer data in the form of the differential equations describing mass and energy balances as a more basic technique for treating many problems now being approached only through dimensional analysis.

\section{NOTATION}

$$
\begin{aligned}
A= & \text { area } \\
a= & \text { one-half the clearance between } \\
& \text { parallel plates } \\
a_{l}= & \text { liquid depth } \\
C_{0}= & \text { concentration of the inlet gas to } \\
& \text { the test section }
\end{aligned}
$$

$C w=$ concentration of the gas at the liquid surface

$c=$ concentration, moles per unit volume

$\bar{c}=$ dimensionless concentration, $\left(c-c_{w}\right) /\left(c_{0}-c_{w}\right)$

$c_{p}=$ heat capacity

$D_{v} \quad=$ molecular diffusivity

$d=$ characteristic diameter

$E \quad=$ eddy diffusivity, $\left(\right.$ distance) ${ }^{2}$ per unit time

$g_{c}=$ conversion factor, $(32.2$ pounds mass/pound force)(feet/second) ${ }^{2}$

$G_{m}=$ flow rate, moles per unit time per

$h=$ heat transfer coefficient

$j=$ a modified $j$ factor for mass transfer

$j_{D}=$ the $j$ factor for mass transfer defined by Colburn, $\left(k_{\diamond} p_{B m} S c^{2 / 3}\right) /$ $G_{m}$ unit area $k \quad=$ thermal conductivity, B.t.u. per unit time per unit area per degree Fahrenheit per foot

$k_{c}=$ mass transfer coefficient, moles per unit time per unit area per unit concentration difference

$k_{a}=$ mass transfer coefficient, moles per unit time per unit area per unit pressure difference

$k_{T}=$ a constant

$N_{A}=$ mass transfer rate, moles per unit time per unit area

$P \quad=$ total pressure

$p=$ gas partial pressure

$p_{B m}=$ logarithmic mean partial pressure of component $B$

$p_{i} \quad=$ gas partial pressure at point $i$

$Q=$ heat flow rate, B.t.u. per unit time

$q \quad=$ heat flow rate, B.t.u. per unit time per unit area

$R \quad=$ the gas constant

$R e=$ the Reynolds number

$S c=$ the Schmidt number

$T=$ temperature

$t=$ time

$U_{a v g}=$ average gas velocity in direction of flow

$U_{\max }=$ maximum gas velocity in direction of flow

$u=$ component of gas velocity in $x$ direction

$V_{1}=$ kinematic viscosity of liquid

$v=$ component of gas velocity in $y$ direction

$w \quad=$ component of gas velocity in $z$ direction

$x=$ coordinate axis; distance

$y / y=y / 2 a=$ coordinate axis; distance

$\epsilon_{\mathrm{c}}=$ eddy conductivity, (distance) ${ }^{2}$ per unit time

$\epsilon_{m}=$ eddy viscosity, $\left(\right.$ distance) ${ }^{2}$ per unit time

$\lambda=$ kinematic viscosity, (distance) ${ }^{2}$ per unit time

$\mu \quad=$ absolute viscosity

$=$ density

$=$ shear stress

\section{LITERATURE CITED}

1. Cairns, R. C., and G. H. Roper, Chem. Eng. Sci., 3, 97 (1954).

2. Colburn, A. P., and T. H. Chilton, Ind. Eng. Chem., 26, 1183 (1934).

3. Corcoran, W. H., F. Page, W. G. Sehlinger, and B. H. Sage, ibid., 44, 410 (1952).

4. Deissler, R. G., Natl. Advisory Comm. Aeronaut. Tech. Note 3145, 15 (1954).

5. Tek, M. R., Ph.D. thesis, Univ. Mich., Ann Arbor (1953).

6. Westkaemper, L. E., Ph.D. dissertation, Univ. Mich., Ann Arbor (1955).

7. Wilke, C. R., and L. A. Bromley, Ind. Eng. Chem., 43, 1641 (1951).

8. Woertz B. B., and T. K. Sherwood, ibid., 31, 1034 (1939).

9. Yuan, S. W., and A. B. Finkelstein, "General Proceedings of Heat Transfer and Fluid Mechanics Institute," Los Angeles, Am. Soc. Mech. Engrs. (1955). 